Anales de Geografía de la Universidad Complutense ISSN: 0211-9803

http://dx.doi.org/10.5209/AGUC.60469

\title{
Detección de barrios vulnerables a partir de la accesibilidad a los servicios públicos de proximidad. El caso de la Ciudad de Valencia ${ }^{1}$
}

\author{
María-Dolores Pitarch-Garrido ${ }^{2}$; Julia Salom Carrasco ${ }^{3}$; Félix Fajardo Magraner ${ }^{4}$ \\ Recibido: 19 de diciembre del 2016 / Enviado a evaluar: 5 de junio del 2017 / Aceptado: 14 de diciembre del 2017
}

Resumen. El análisis de la accesibilidad a los servicios públicos de proximidad es una manera de acercarnos al estudio de la sostenibilidad social en las ciudades. En este artículo se analizacómo la localización de dichos servicios (centros de especialidades médicas, institutos de enseñanza secundaria y servicios sociales de base) contribuye a mejorar la calidad de vida de las personas. Para ello se ha estimado la accesibilidad desde el lugar de residencia al centro de oferta de servicio en la ciudad de Valencia utilizando SIG e información espacialmente detallada (por número de policía o portal). La relación entre dicha accesibilidad y una serie de variables de tipo socioeconómico facilita la detección de barrios con peores dotaciones sociales y permite caracterizar dichas áreas según su estructura socioeconómica y población en riesgo de exclusión, con el fin de facilitar la toma de decisiones para la intervención pública.

Palabras clave: Accesibilidad; servicios públicos; riesgo de exclusión; sostenibilidad social; Valencia.

1 Este artículo se ha elaborado en el marco del proyecto "Sostenibilidad social, conectividad global y economía creativa como estrategias de desarrollo en el Área metropolitana de Valencia" (CSO2016-74888-C4-1-R), financiado por la Agencia Estatal de Investigación (AEI) y al Fondo Europeo de Desarrollo Regional (FEDER) dentro del Programa Estatal de Investigación, Desarrollo e Innovación Orientada a los Retos de la Sociedad, en el marco del Plan Estatal de Investigación Científica y Técnica y de Innovación 2013-2016, convocatoria de 2016.Uno de los autores, Félix Fajardo, cuenta con una ayuda para contratos predoctorales para la formación de profesorado universitario, convocatoria 2014 (FPU14/05550), financiada por el Ministerio de Educación, Cultura y Deporte.Una primera versión de este artículo en formato de comunicación se presentó al XIII Congreso de Geografía Urbana celebrado en Girona entre los días 16 y 18 de junio de 2016.

2 Departamento de Geografía. Instituto Interuniversitario de Desarrollo Local.

E-mail: maria.pitarch@uv.es

3 Departamento de Geografía. Instituto Interuniversitario de Desarrollo Local.

E-mail: julia.salom@uv.es

4 Departamento de Geografía. Instituto Interuniversitario de Desarrollo Local.

E-mail: felix.fajardo@uv.es 


\title{
[en] Detection of areas in risk of exclusion from the accessibility to public services of proximity. The case of the city of Valencia.
}

\begin{abstract}
The analysis of the accessibility to the proximity public services is a way of approaching the study of social sustainability in cities. In this article it is analyzed how the location of the above mentioned services (centers of medical specialities, centers of secondary education and basic social services) helps to improve the quality of life of people. The accessibility has been estimated from the place of residence to the center of supply in the city of Valencia using GIS and information spatially detailed -for each building-. The relationship between this accessibility and a series of socioeconomic variables facilitates the detection of neighborhoods with worse social endowments and allows characterizing these areas according to their socioeconomic structure and population at risk of exclusion, in order to facilitate decision-making for the public intervention.
\end{abstract}

Keywords: Accessibility; public services; risk of exclusion; social sustainability; Valencia.

\section{[fr] Détection des zones à risque d'exclusionà partir de l'accessibilité auxservices publics de proximité. Le cas de la ville de Valencia.}

Résumé. L'étude de l'accessibilitéauxservicespublics de proximitéest une approche d'analyse de la soutenabilitésociale desvilles. Cetarticle a pourbutd'analyserl'effet de la localisation des servicessusmentionnés (centres de spécialitésmédicales, centres d'enseignementsecondaire et servicessociaux de base) sur l'amélioration de la qualité de vie des personnes. L'accessibilité a étéestimée à partir du lieu de résidenceau centre d'approvisionnement de la ville de Valence à l'aide de SIG et des informationsspatialementdétailléespourchaquebâtiment. La relation entre cetteaccessibilité et une série de variables socio-économiques facilite la détection des quartiersurbains et des zones les plus desservies et permets de caractériser les quartiers en fonction de leurstructure socio-économique et de leurniveau de risque de pauvreté. Ceci facilite la prise de décision des responsables publics en la matière.

Mots clés: Accessibilité; servicespublics; risque d'exclusion; soutenabilitésociale; Valencia.

Cómo citar. Pitarch-Garrido, M.D., Salom Carrasco, J. y Fajardo Magraner, F. (2018): Detección de barrios vulnerables a partir de la accesibilidad a los servicios públicos de proximidad. El caso de la Ciudad de Valencia. Anales de Geografia de la Universidad Complutense, 38(1), 61-85.

Sumario. 1. Introducción: equipamientos públicos y ciudad. 2. Objetivos y metodología. 3. Resultados. 3.1. Delimitación de áreas según accesibilidad a los servicios de proximdad en la ciudad de Valencia. 4. Conclusiones. 5. Bibliografía.

\section{Introducción: equipamientos públicos y ciudad}

Los servicios y equipamientos públicos han sido objeto de estudio desde la academia por parte de diversas ciencias, incluida la Geografía. La transformación de las sociedades occidentales en economías de servicios y la aparición y consolidación del Estado del Bienestar se encuentran en la base de este interés creciente por una serie de actividades extraordinariamente heterogéneas en cuanto a tipología y a comportamiento espacial.

Los servicios públicos -y los equipamientos a ellos asociados- resultan de especial interés para los geógrafos por sus dos características esenciales: en primer lugar, son 
una vía directa para la redistribución espacial y social de las rentas públicas; y, en segundo lugar, como consecuencia de lo anterior, su oferta por parte de la administración pública los convierte en instrumentos de las políticas de desarrollo económico, social y territorial. Como indican Calvo et al (2001), los servicios públicos juegan un "importante papel en el desarrollo regional y (...) son hoy día en nuestra sociedad uno de los motores esenciales del cambio, lo que obviamente se traduce en que una buena disposición de los mismos va, inevitablemente, a acabar revirtiendo en el desarrollo regional." (p. 251).

Durante el periodo de crisis económico-financiera actual en España, la política tanto del gobierno central como de los gobiernos regionales ha tendido hacia la restricción del gasto público. Esta política de austeridad ha supuesto recortes en las inversiones dirigidas a aumentar los recursos y equipamientos públicos. Dado que una de las características de la exclusión es la imposibilidad o dificultad de las personas excluidas para acceder a los mecanismos de protección social, entre ellos, los servicios públicos básicos, las políticas de austeridad en tiempos de crisis no han hecho sino acrecentar la tensión y la desigualdad social (Subirats, 2005).

El análisis de los contrastes socio-espaciales internos a las ciudades y áreas metropolitanas constituye un tema fundamental en la tradición epistemológica de la Geografía Urbana. El espacio urbano es una de las arenas en las que tienen lugar los procesos espaciales asociados a la crisis económica y donde, en palabras de Fernández Durán (1993), la distancia social entre los grupos privilegiados y los excluidos es mucho mayor que la distancia física que los separa. En este entorno global aparecen nuevas formas de marginalidad y polarización. Según Sassen (1991), "la evidencia, para el caso de Estados Unidos, Europa Occidental y Japón, sugiere que será necesaria una acción y política gubernamentales para reducir las nuevas formas de inequidad espacial y social." (p. 8).Una de las formas en las que la política puede actuar para paliar las situaciones de pobreza y exclusión social es a través de la planificación de la oferta pública de servicios. En toda planificación la localización del equipamiento y las posibilidades de acceso al mismo resultan básicas para una adecuada gestión del territorio a nivel local.

Uno de los condicionantes de la eficacia y eficiencia de los servicios públicos es la elección de un buen emplazamiento, lo cual se encuentra implícitamente vinculado a una buena accesibilidad. La distribución espacial de las actividades económicas en ocasiones es irregular y desequilibrada, lo cual dificulta la consecución de uno de los principiosque caracterizan dichos servicios: la universalidad.

Además de este principio ético de universalidad, asociado a una localización óptima y a unos presupuestos infinitos, lo cual no deja de ser una utopía, los servicios públicos deben localizarse cerca de los usuarios, cualquiera que sea su nivel económico o social. Los serviciosde proximidad, los más frecuentemente demandados por la población, suponen un determinado uso del tiempo con implicaciones en la calidad de vida (Marquet y Miralles, 2014). Las altas densidades de población unidas a una extensa oferta de transporte público y a un espacio urbano caracterizado por una determinada mezcla de usos, son factores que contribuyen de manera trascendente a 
la configuración de un espacio urbano equilibrado y menos proclive a la aparición de espacios de exclusión.

La actual coyuntura de crisis económica, que supone una menor disponibilidad de recursos públicos, implica, entre otras cosas, la necesidad de gestionar con eficacia los recursos disponibles y de introducir fórmulas innovadoras de gestión que posibiliten -ahora más que nunca- una mejora de la calidad de vida de la población. Durante los periodos de recesión económica, las personas reducen sus gastos y, entre ellos el de transporte, incluido el público, lo cual significa que aumentan los desplazamientos a pie (Albertos,2014); y,ya que los servicios básicos(educación, sanidad y servicios sociales)son siempre demandados,y muy especialmente en estos años de crisis (servicios sociales), su cercanía resulta fundamental para garantizar su universalidad real.

En estas circunstancias, y dado que la ciudad es el lugar en el que la justicia y la equidad quedan más claramente definidas (Sassen, 1991, 1998), el impacto de la crisis sobre las familias, unido a una política de restricciones presupuestarias, explica la aparición de nuevos -y la consolidación de los viejos- espacios de exclusión en las ciudades. En el interior de las grandes áreas urbanas, por tanto, existen barrios que, por sus características y circunstancias, son marginales; y otros que-como señalaron ya Benko y Lipietz (1994) respecto a las regiones- ganan, es decir, que salen beneficiados de la consolidación de una situación producto de políticas y decisiones públicas. Es, pues, la ciudad, desde una perspectiva local, el espacio más adecuado para definir los procesos de mejora en la equidad y, por tanto, para trabajar por la consecución de una mayor justicia espacial.

En un mundo global en el que la pobreza y la desigualdad crecen, se ha extendido el concepto de justicia espacial que, aparte de ser un fenómeno social, incorpora también el aspecto geográfico de la misma, ya que las injusticias ocurren en espacios concretos. Se trata, pues, de una categoría de análisis que contribuye a entender e interpretar la realidad territorial y social a partir del análisis de las condiciones que originan las injusticias, en particular aquellas relacionadas con la distribución de la riqueza en forma de bienes, servicios y oportunidades. La definición de justicia está muy vinculada a la de equidad. Como señala Rawls (2001), la justicia como equidad incluye un concepto político de justicia, pues no es natural al ser humano; por tanto, forma parte de la estructura básica de la que se dota la sociedad, es decir, del pacto social. Es un derecho básico de todos los seres humanos, sea cual sea su condición, pero no es absoluto ni inamovible, sino que debe ser negociado como un punto intermedio entre la libertad personal y la igualdad, punto que debe definirse como producto de un acuerdo entre los agentes sociales implicados, y que, por tanto, puede variar dependiendo de la sociedad. En las sociedades occidentales, el desarrollo de sistemas de bienestar que garantizan la universalidad de los derechos ciudadanos básicos, es decir, la equidad espacial, es objeto prioritario de toda política pública; y la implicación en la misma de los diferentes actores, así como la consideración de las condiciones socioeconómicas y culturales de cada territorio, tienen consecuencias sobre el desarrollo, la equidad y la cohesión social (Hernández Aja, 2000; Andreotti, 
Mingione y Polizzi, 2012, Alguacil, Camacho, y Hernández Aja, 2014; PitarchGarrido, 2018).

En este sentido, resulta relevante insistir en la importancia del análisis del territorio a nivel local, a escala de ciudad y de barrio. La mayoría de las investigaciones geográficas empíricas se plasman en textos de alcance regional y, sobre todo en Geografía Urbana, local. Es en esta escala donde los geógrafos pueden "subrayar la diferencia geográfica y los factores que la sustentan y la perpetúan" (Sánchez Hernández, 2003, p. 166). La globalización y los debates sobre naciones y bloques se trabajan desde concepciones intelectuales construidas a partir de los resultados obtenidos en investigaciones a escala local. Las redes globales (por ejemplo, de ciudades) se sustentan en nodos locales donde las lógicas del mercado y las acciones políticas están más próximas a la realidad. La atención de la Geografía Urbana a partir de la crisis del modelo económico fordista se ha centrado más en el análisis de las regiones-problema, en un claro interés por los procesos de cambio espaciales en el interior de las ciudades. Estos cambios se producen insertos en un proceso dinámico de transformación y competencia en estos espacios, por lo que su análisis sigue siendo muy pertinente. El objetivo de toda investigación social es la mejora de la calidad de vida de las personas, para lo cual la lucha contra la desigualdad resulta crucial, y, para ello, los estudios de caso pueden aportar experiencias, métodos $\mathrm{y}$ formas de intervención que permitan aumentar el conocimiento sobre estos espacios y que, además, sean transferibles a otros territorios. Este tipo de análisis es abundante en la literatura de las ciencias sociales y de la Geografía en países anglosajones y europeos (Sassen, 1998, Werna, 2001, Harvey, 2012, Hudson, 2013, Sheltonet al. 2015, Bloklandet al., 2016), y también en España (Calvo et al. 2001, Nel.lo, 2007, Marquet y Miralles, 2014,Cerdá, 2014, Pitarch, 2013, Salom y Fajardo, 2015).

Un aspecto más a considerar es la política urbana. Desde los años 80 del pasado siglo en algunas ciudades europeas y españolas se han puesto en marcha políticas de desarrollo cuya idea central es que los gobiernos locales deben ser más innovadores y empresariales. Para ello se utilizan dos estrategias fundamentales: la intervención directa del gobierno en la puesta en marcha de nuevas empresas, o la creación de un entorno que propicie el desarrollo de actividades económicas innovadoras con el fin de recuperar la competitividad perdida. Es lo que se conoce como New Economic Policy, que trata de reorientar el papel del Estado para convertirlo enejecutor de infraestructuras que apoyen, financien o promuevan nuevas formas de acumulación de capital a partir del uso de los recursos públicos. Harvey (2012) denomina a esta situación "urbanización del capital" y "rentas de monopolio", lo que, en resumen, supone que las características de un lugar, concebidas o construidas como únicas e irrepetibles, consiguenaumentarel valor de ese territorio, ciudad o barrio. Con el fin re-imaginar y recrear el espacio urbano, sobre todo para el posible inversor o el turista adinerado, se desarrollan estrategias no únicamente económicas, sino orientadas a la gestión de la dimensión simbólica y la construcción de identidades. Así, el citymarketing y el brand management entran en la política local, y los procesos de 
espectacularización vinculados a los eventos y grandes proyectos contribuyen a la creación de la marca urbana. La puesta en marcha de políticas urbanas basadas en el brand management y el city marketing en la ciudad de Valencia entre los años 1995 y 2015 la ha convertido en paradigma de la política urbana neoliberal.A finales de los 80 , pero sobre todo en los años 1990 , con un gobierno regional y municipal de perfil conservador, el objetivo político será conseguir una nueva imagen internacional a partir de grandes construcciones emblemáticas y de la organización de eventos deportivos y sociales a nivel internacional Algunos autores señalan que este proceso ha convertido a la ciudad de Valencia en una "metrópoli clónica glocalizada" (Santamaría y Moncusí, 2013). Una de las consecuencias de esta política fue la construcción de áreas nuevas con el fin de mejorar los espacios obsoletos, a lo que se une la aparición de espacios residenciales de élite junto a barrios tradicionales con problemas propios, y la promoción de centros comerciales en la periferia metropolitana. Las principalesconsecuencias de la megalomanía de los gobiernos valencianos hasta la llegada de la crisis económica se resumen en el aumento de los precios de la vivienda,la pérdida del espacio de huerta alrededor de la ciudad, la dificultad del acceso a la vivienda o a los servicios básicos (por escasos o lejanos), etc. Durante los años en los que en Valencia se invertía (y endeudaba) en la construcción de una nueva ciudad, entre 1998 y 2008, el valor catastral de los inmuebles aumentó más de un 65\% (Salom y Pitarch, 2017), mientras que la renta familiar disponible se redujo considerablemente, lo que evidencia una mayor dificultad en el acceso a la vivienda. A ello se suma el hecho de que una parte sustancial de los fondos europeos para el desarrollo regional se destinóa los grandes proyectos urbanísticos y las infraestructuras que les acompañaron (Monfort, 2010, Gaja, 2016), lo que supuso un coste de oportunidad importante, pues dejó de invertirse en otros equipamientos que pudieran haber sido esenciales para la mejora de la calidad de vida de algunos barrios (Salom y Pitarch, 2017). El modelo seguido por Valencia ha buscado el desarrollo económico-empresarial, con dudoso éxito pero importantes consecuencias, como la especulación, el beneficio de unos pocos -sobre todo las empresas constructoras-, y la agudización de los desequilibrios sociales y espaciales.

El caso de estudio que aquí presentamos es la ciudad de Valencia, cuya historia más reciente, tamaño y características socioeconómicas la definen como una realidad compleja de gran interés para su análisis, así como para la acción pública (políticas de planificación espacial) cuyo fin es la mejora de las condiciones de vida de las personas que la disfrutan.

\section{Objetivos y metodología}

El objetivo principal de este artículo es desarrollar y aplicar una metodología para la detección de áreas de acción prioritaria a partir del análisis de la accesibilidad a los servicios públicos y las características sociodemográficas de la población. Así, es posible conocer si la oferta de servicios de proximidad en la ciudad de Valencia responde a la demanda actual (postcrisis) y contribuye a paliar los desequilibrios 
asociados a la estructura socioeconómica de los barrios o áreas de la ciudad, en particular de aquellos más degradados desde el punto de vista socioeconómico, o, por el contrario, es ineficaz o incluso contribuye a agravar las desigualdades.

Con este fin, se ha trabajado sobre la ciudad de Valencia, un municipio con 787.266 habitantes en 2015, 156.835 de ellos mayores de 65 años, 116.615 menores de 16 años, 94.050 residentes extranjeros, y una tasa de paro que alcanzaba el $18,9 \%$ en el cuarto trimestre de 2015.

El análisis de la accesibilidad a los equipamientos públicos está justificado por la importancia que los servicios asociados a éstos tiene como vía de redistribución social y espacial de las rentas públicas, así como por las posibilidades que aportan para las política de gestión territorial y mejora de la calidad de vida. Los servicios públicos que se han considerado para este análisis han sido los siguientes: Centros de Especialidades Médicas (del Servicio Valenciano de Salud), los Institutos de Educación Secundaria (públicos y concertados), y los Servicios Sociales de base, dependientes de la administración local y primer escalón para la inclusión de la persona en el sistema de apoyo social público.

El cálculo de la accesibilidad a los servicios seleccionados se ha realizado a partir de la localización de la población en su lugar de residencia, es decir, el portal o número de policía que figura en el Padrón de Habitantes de 2014. Dicha información, tan detallada desde el punto de vista espacial (incluye el número de personas con algunas de sus características como edad, nivel de formación y procedencia), ha sido cedida, totalmente anonimizada, por la Oficina de Estadística del Ayuntamiento de esta ciudad. Esto nos ha permitido obtener unos resultados muy fiables, con un detalle preciso. Para preservar el secreto estadístico, los datos de las viviendas en donde el número de personas empadronadas era inferior a 4 han sido agregados al portal inmediatamente anterior o posterior; seleccionando en todos los casos el número con menor población empadronada. Si aún a pesar de la anterior agregación, la resultante sigue siendo inferior a 4 personas, se ha repetido el procedimiento anterior, hasta obtener un agregado que cumplía el criterio. Las calles en las que, a pesar de aplicar las reglas de agregación anteriores, no se logra obtener un agregado con 4 o más personas, se han marcado como calles con 3 o menos habitantes empadronados, sin clasificar por ninguna de las variables demográficas, todo ello con el fin de mantener el secreto estadístico. De cualquier forma, esto afecta a un porcentaje pequeño de los hogares valencianos, y el error de localización con respecto a los objetivos de este proyecto es mínimo.

Para medir la accesibilidad se han considerado dos posibilidades: a pie o en transporte público. Dado que los servicios seleccionados no presentan una oferta tan amplia como otros, la posibilidad de trasladarse en transporte público también ha sido considerada. No se considera el transporte privado, pues éste sí que supone desigualdades y, por sus características de uso, no es el más adecuado para el acceso a los servicios de proximidad, ni resulta sostenible para una ciudad compacta como es Valencia. 
En cuando a la medida de la accesibilidad, hemos optado, de entre el amplio abanico de índices de existente (Garrocho y Campos, 2006; Bhatet al. 2000), por calcular el índice de separación espacial,cuya medida en minutos resulta fácilmente interpretable y útil. El índice se ha calculado desde cada portal al punto de oferta más cercano de cada tipo de servicio público. A partir del análisis de la accesibilidad, es posible detectar, mediante la cartografía y las tablas resultantes, territorios en los que se concentran determinadas características dotacionales con el fin de conocer si existen fenómenos de polarización espacial.

En este tipo de índice todos los puntos de origen tienen el mismo peso en los cálculos, y el índice sólo recoge la información referente a las distancias. La complejidad la introduce el uso de la red de movilidad real con tiempos reales contrastados.

Según esto, el Índice de Separación Espacial de la unidad espacial $i\left(I S E_{i}\right)$ es igual a:

$$
\begin{aligned}
& I S E=\sum D_{i j} / n \\
& \text { donde } \\
& i \text { es el lugar de origen (portales) para el que se calcula el índice } \\
& j \text { es cada uno de los potenciales lugares de destino de los desplazamientos } \\
& \quad \text { (equipamientos). Sólo se utiliza el más cercano al lugar de origen. } \\
& D_{i j} \text { es la distancia en minutos, -obtenida a partir de las matrices calculadas-, } \\
& \quad \text { entre el lugar de origen i y el lugar de destino } \mathrm{j} \\
& n \text { es el número de posibles lugares de destino. }
\end{aligned}
$$

En la ciudad de Valencia hay un total de 25.870 puntos de demanda y, para cada tipo de servicio, un número elevado de puntos de oferta.Ello requiere el uso de tecnología SIG para poder trabajar con matrices tan amplias. Se han utilizado dos programas informáticos: TRANSCAD 6.0 y ARCINFO 10.0. A partir de las bases de datos de Tele-Atlas (vías) y de la información cedida por la Oficina Estadística del Ayuntamiento de Valencia, se ha construido un SIG para la ciudad con la localización de todos los servicios y toda la red de transporte público, según tipo: metro-tranvía, autobús urbano, autobús metropolitano y tren de cercanías. Se cuenta también, evidentemente, con la posibilidad de realizar el trayecto a pie.

Se ha realizado una significativa labor de depuración de la información y de generación de topología (corrección de errores y conexión de portales a los nodos de la red), cálculo de velocidades, agregación de información adicional como los horarios del transporte público (frecuencia), velocidades medias para cada línea, tiempos de espera, etc.

El modelo utilizado implica el cálculo de la movilidad urbana a través de las redes por las que se desarrolla, utilizando para ello el programa TRANSCAD 6.0. que posibilita la inclusión de elementos fundamentales como son los tiempos de acceso al transporte público, de espera, de transbordo entre modos, y de desplazamiento efectivo. 
La expresión que emplea TRANSCAD para la determinación del tiempo y a partir de la cual se creará el camino mínimo será la siguiente:

$$
C G=V_{*}\left(p_{v^{*}} T_{v}+p_{e i *} T_{e i}+p_{e t^{*}} T_{e t}+p_{c^{*}} T_{c a}+p_{c^{*}} T_{c e}+p_{c^{*}} T_{c t}+p_{p^{*}} T_{p}+p_{t^{*}} T_{t}\right)+N * C_{b}
$$

donde

$T$ hace referencia a los tiempos, de modo que:

$T_{v}$ es el tiempo recorrido en vehículo (en un transporte público).

$T_{e i}$ es el tiempo de espera inicial (para acceder al transporte público). TransCAD lo hace depender de la frecuencia de paso.

$T_{e t}$ es el tiempo de espera debido a un trasbordo.

$T_{c a}$ es el tiempo recorrido a pie para acceder al transporte público.

$T_{c e}$ es el tiempo recorrido a pie al abandonar el transporte público.

$T_{c t}$ es el tiempo recorrido a pie para realizar el trasbordo entre transportes públicos.

$T_{p}$ es el tiempo invertido en las paradas. Dependerá del número de paradas realizadas $\left(N_{p}\right)$ y del tiempo invertido en cada una de ellas $\left(T_{p l}\right)$.

$T_{t}$ es la penalización (en tiempo) por trasbordo.

$p$ hace referencia a los pesos aplicados sobre dichos tiempos (nótese que el peso aplicado a todos los recorridos a pie - acceso, trasbordo y egreso- es el mismo).

$V$ es el coste monetario equivalente de un minuto de viaje y permite la conversión del tiempo de viaje a coste generalizado (en este caso monetario).En este caso se ha considerado como 0.

$C_{b}$ es el coste de cada billete y $N$ el número de billetes que es necesario adquirir. En este caso se ha considerado como 0 .

Una vez calculada la accesibilidad a pie y en transporte público para cada uno de los tres tipos de oferta pública, considerada desde cada uno de los puntos de origen, se ha procedido a transformar los datos puntuales en superficies a partir de la interpolación mediante el método IDW (Inverse Distance Weighting), utilizando la herramienta correspondiente del módulo Spatial Analysis de ARCINFO 10.0. El IDW considera que los datos están correlacionados en el espacio, por lo que es capaz de estimar los valores superficiales a partir de datos de puntos asignando pesos a los valores del entorno en función inversa de la distancia que los separa (Cañada Torrecilla, 2007). En nuestro caso, este método se ha aplicado a los valores puntuales de ISE utilizandoun exponente de distancia de2, un radio de búsqueda delos 12 puntos más cercanos entre sí (separados por una distancia máximade500 metros), y un tamaño de celda de $25 \times 25 \mathrm{~m}$. Sobre la superficie así estimada, se han delimitado áreas con niveles que consideramos significativos en relación con la accesibilidad. Se han seleccionados tres umbrales para dos formas de movilidad: a pie o en transporte público. Para la primera se han considerado los tiempos menores o iguales a 10 minutos, de 10 a 20 minutos y más de 20 minutos. Para la segunda, los tiempos son 
algo más amplios: menos o igual a 20 minutos, de 20 a 40 minutos y más de 40 minutos. Los intervalos de tiempo considerados pueden variar para ajustarse a distintos escenarios.

Por último, con el fin de poder comparar las características físicas, sociales y económicas de los barrios de la ciudad con los datos de accesibilidad, se ha procedido a agrupar (sumar) la información sociodemográfica a nivel de portal, a partir de una malla de polígonos cuadrados de 100 metros de lado inscrita sobre la ciudad de Valencia. El objetivo es tener una agrupación de información a una escala intermedia, entre el barrio (excesivamente amplio) y el portal (difícil de representar por la multitud de puntos que supone y potencialmente afectado por fenómenos de aleatoriedad). Además de las características de su estructura sociodemográfica (procedentes de la información del Padrón por portal, indicadores medios de cada cuadrado), se han asociado a cada cuadrado distintos indicadores de la situación económicadel barrio en que se ubican, así como la información sobre accesibilidad a los servicios básicos a partir del cálculo del ISE medio para cada cuadrado, servicio y tipo de transporte.La información utilizada para la caracterización económica de cada área procede dediversas fuentes, entre las que destacan el Catastro (2014), el Padrón (2014) y el Ayuntamiento de Valencia (Impuesto de Actividades Económicas e impuesto de vehículos de tracción mecánica -Parque de Vehículos-) (ver tabla 1).

Para simplificar y estructurar la información inicial, se ha realizado un Análisis Factorial de Componentes Principales (ACP). Este método permite reducir el volumen de los datos transformando un conjunto de variables iniciales en otro conjunto de variables correlacionadas entre sí llamadas Componentes Principales (Foguet, 1989). De este modo, es posible determinar los factores subyacentes comunes a grupos de variables asociadas entre sí. En el presente caso se ha aplicado un análisis factorial mediante el método de Componentes Principales a las 13 variables seleccionadas a partir del marco teórico y de los resultados de la matriz de correlaciones. El tratamiento estadístico se ha realizado con el programa IBM SPSS Statistics, Versión 22. El análisis ha dado resultados significativos, según el test Bartlett (nivel de significación inferior al 0,001) y el índice Kaiser-Meyer-Olkin $(\mathrm{KMO}=0,697)$. También se ha realizado una rotación a la matriz mediante el método VARIMAX a fin de identificar con mayor claridad la relación que se establece entre los factores y las variables y facilitar la interpretación de los resultados. 
Tabla 1. Variables seleccionadas para el análisis factorial.

\begin{tabular}{|c|c|c|}
\hline INDICADOR & VARIABLE & FUENTE \\
\hline $\begin{array}{l}\text { Estructura } \\
\text { por edades }\end{array}$ & $\begin{array}{c}\text { Porcentaje } 65 \text { y más años } \\
\text { Porcentaje menores de } 16 \text { años }\end{array}$ & Padrón 2014 \\
\hline $\begin{array}{l}\text { Nivel de } \\
\text { estudios }\end{array}$ & $\begin{array}{c}\text { Porcentaje de población de } 24 \\
\text { y más años con menos de Bachiller }\end{array}$ & Padrón 2014 \\
\hline $\begin{array}{l}\text { Situación } \\
\text { económica } \\
\text { de las } \\
\text { familias }\end{array}$ & $\begin{array}{c}\text { Edad media de las viviendas } \\
\text { Valor por m² de la vivienda } \\
\text { Porcentaje de vehículos con menos de } 80 \mathrm{CV} \text { sobre el total } \\
\text { de vehículosdel barrio } \\
\text { Porcentaje de vehículos con más de } 200 \mathrm{CV} \text { sobre el total de } \\
\text { vehículos del barrio } \\
\text { Porcentaje de empresas e instituciones financieras y seguros } \\
\text { sobre el total de empresas del barrio } \\
\text { Porcentaje de empresas de comercio, restaurantes, hostelería } \\
\text { y reparaciones sobre el total de empresas del barrio } \\
\text { Porcentaje de empresas profesionales sobre el total de } \\
\text { empresas del barrio } \\
\text { Porcentaje ocupados en comercio, hostelería y transportes } \\
\text { sobre el total de ocupados residentes en el barrio } \\
\text { Porcentaje de ocupados en finanzas, derecho y seguros } \\
\text { sobre el total de ocupados residentes en el barrio }\end{array}$ & $\begin{array}{c}\text { Oficina de } \\
\text { Estadística del } \\
\text { Ayuntamiento de } \\
\text { Valencia: Catastro } \\
\text { (2014), Censo de } \\
\text { Actividades } \\
\text { Económicas } \\
\text { (2015) } \\
\text { Parque de } \\
\text { Vehículos (2015) }\end{array}$ \\
\hline Nacionalidad & Porcentaje de nacionalidad extranjera extracomunitaria & Padrón 2014 \\
\hline
\end{tabular}

Fuente: Elaboración propia.

Tabla 2. Varianza total explicada para los factores con autovalores superiores a 1para la determinación de los Componentes Principales

\begin{tabular}{|c|c|c|c|c|c|c|c|c|c|}
\hline \multirow[b]{2}{*}{ Componente } & \multicolumn{3}{|c|}{ Autovalores iniciales } & \multicolumn{3}{|c|}{$\begin{array}{l}\text { Sumas de extracción de cargas al } \\
\text { cuadrado }\end{array}$} & \multicolumn{3}{|c|}{$\begin{array}{l}\text { Sumas de rotación de cargas al } \\
\text { cuadrado }\end{array}$} \\
\hline & Total & $\begin{array}{l}\% \quad \mathrm{de} \\
\text { varianza }\end{array}$ & $\begin{array}{l}\% \\
\text { acumulado }\end{array}$ & Total & $\begin{array}{l}\% \text { de } \\
\text { varianza }\end{array}$ & $\begin{array}{l}\% \\
\text { acumulado }\end{array}$ & Total & $\begin{array}{l}\% \text { de } \\
\text { varianza }\end{array}$ & $\begin{array}{l}\text { \% } \\
\text { acumulado }\end{array}$ \\
\hline 1 & 4,813 & 40,108 & 40,108 & 4,813 & 40,108 & 40,108 & 3,917 & 32,644 & 32,644 \\
\hline 2 & 1,708 & 14,237 & 54,345 & 1,708 & 14,237 & 54,345 & 2,099 & 17,489 & 50,133 \\
\hline 3 & 1,246 & 10,387 & 64,732 & 1,246 & 10,387 & 64,732 & 1,630 & 13,586 & 63,719 \\
\hline 4 & 1,005 & 8,371 & 73,103 & 1,005 & 8,371 & 73,103 & 1,126 & 9,384 & 73,103 \\
\hline 5 & 0,744 & 6,199 & 79,302 & & & & & & \\
\hline
\end{tabular}

Fuente: Elaboración propia. 
Tabla 3. Matriz de Componente Rotado. Componente 1. Método de rotación: VARIMAX con normalización Kaiser

\begin{tabular}{|l|c|}
\hline & Componente 1 \\
\hline Porcentaje de menores de 16 años &, 048 \\
\hline Porcentaje 65 y más años &,- 089 \\
\hline Porcentaje nacionalidad extranjera extracomunitarios &,- 086 \\
\hline Porcentaje de empresas profesionales &, 796 \\
\hline $\begin{array}{l}\text { Porcentaje de empresas de comercio, restaurantes, } \\
\text { hostelería y reparaciones }\end{array}$ &,- 851 \\
\hline $\begin{array}{l}\text { Porcentaje de empresas e instituciones financieras y } \\
\text { seguros }\end{array}$ &, 847 \\
\hline $\begin{array}{l}\text { Porcentaje de ocupados en finanzas, derecho y } \\
\text { seguros }\end{array}$ &, 048 \\
\hline Edad media de las viviendas &,- 669 \\
\hline Porcentaje de vehículos con menos de 80 CV &, 198 \\
\hline Porcentaje de vehículos con más de 200 CV &, 585 \\
\hline $\begin{array}{l}\text { Porcentaje de población de 24 y más años con menos } \\
\text { de Bachiller }\end{array}$ &,- 600 \\
\hline Valor por m ${ }^{2}$ de la vivienda &, 796 \\
\hline
\end{tabular}

Fuente: Elaboración propia.

Existen varios criterios para determinar el número de componentes a retener. Según López et al (2016) un criterio principal consiste en valorar el contenido de los factores y sus implicaciones en el análisis en cuestión. Atendiendo a este criterio, se ha seleccionado el primer componente, que explica por sí mismo el 32,6\% de la varianza (tabla 2). La matriz de componente rotado (tabla 3 ) muestra las variables que conforman el componente 1, de modo que es posible interpretar el sentido de dicho componente. Como se puede observar, las variables que conforman este componente no se refieren sólo a las características de la población residente (nivel educativo elevado, precio y antigüedad de la vivienda), sino también a la especialización funcional de las áreas (presencia de profesionales y empresas e instituciones financieras). Ello podría conducir a confusión en el caso de que el objetivo fuera identificar las zonas de residencia de la población acomodada, ya que ésta no necesariamente coincide con la localización de los servicios más avanzados, por ejemplo. Sin embargo, ya que en nuestro caso el objetivo es detectar las áreas en peligro de exclusión, el valor del componente en sentido negativo nos da una idea muy ajustada de la localización de la población con menor nivel de renta, que generalmente reside en espacios de menor calidad funcional, caracterizados por bajos precios del suelo (y de la vivienda) y ausencia de funciones superiores.

Por tanto, la comparación de la cartografía de las puntuaciones factoriales de este componente con la de las áreas de baja accesibilidad medidas por el índice ISE para cada tipo de servicio, a pie y en transporte público, nos permite identificar las áreas de 
la ciudad de Valencia donde coinciden un bajo nivel socioeconómico de la población con una lejanía relativa a los servicios públicos de proximidad y, por tanto, laszonas consideradas como de mayor riesgo social, susceptibles, por tanto, de una intervención pública más inmediata.

\section{Resultados}

\subsection{Delimitación de áreas según accesibilidad a los servicios de proximidad en la ciudad de valencia}

La cartografía resultante del análisis de la accesibilidad a cada uno de los servicios analizados (figuras 1, 2 y 3) permite definir las áreas interiores de la ciudad con mejores y peores condiciones en cuanto a tiempo de desplazamiento.

De los tres tipos de servicios considerados, son los institutos de educación secundarialos que presentan una localización más dispersa sobre el territorio, por lo que su accesibilidad a pie es bastante buena, mejorando, sólo ligeramente, en el caso de uso del transporte público. Por el contrario, los centros de especialidades médicas, al ser menos numerosospor tratarse de servicios especializados, muestran una pauta territorial algo más concentrada, que determina distancias más largas a recorrer por parte del usuario, tanto a pie como en transporte público. Por su parte, los servicios sociales de base muestran una situación intermedia, con una clara mejora si se recurre al transporte público, siendo las áreas peor servidas sólo las ubicadas en la periferia urbana.

Las tablas 4 a 6 muestran la población residente en la ciudad de Valencia afectada por peores condiciones de accesibilidad, en términos de tiempo de desplazamiento, a los tres tipos de servicios públicos considerados. En el caso de los centros de especialidades médicas, la accesibilidad a piede una parte importante de la población se sitúa por encima del umbral de 20 minutos. Más de 440.000 personas residentes en la ciudad de Valencia, el 59,2\% del total, se encuentran a más de 20 minutos andando de un centro de especialidades médicas. Esta cifra se reduce considerablemente si se utiliza el transporte público, aunque también resulta muy elevada; casi 15.000 personas (1,9\%del total) se encuentran a más de 40 minutos en transporte público de un centro de especialidades médicas.Llama la atención la gran cantidad de ancianos (mayores de 65 años) que reside a más de veinte minutos andando del servicio, sobre todo si lo comparamos con la cifra de menores de 16 años, lo que nos avanza que son algunos de los barrios más envejecidos los más afectados por una peor accesibilidad.

Las áreas con peores condiciones de accesibilidad a los centros de especialidades médicas se extienden a lo largo de los barrios de la periferia urbana, siendo este problema especialmente relevante en el sur de la ciudad por la mayor cantidad de población afectada; así, el $99 \%$ de la población que reside a más de 20 minutos andando de un centro público de especialidades médicas reside en la mitad sur del casco urbano). En cambio, y aunque la mejora general de la accesibilidad es evidente, 
cuando se utiliza el transporte público las zonas menos accesibles se sitúan al norte de la ciudad. Se trata de un área que incluye los barrios de Massarrojos, Benifaraig, Carpesa, Borbotó y Mauella, que reúne el $26 \%$ de la población de la ciudad que se encuentra a más de 40 minutos del centro de especialidades médicas más cercano. Por el contrario, la cifra de población afectada en el sur, aunque importante, no es tan alta como la hallada cuando consideramos los desplazamientos a pie.

En cuanto a la red de servicios sociales, tan necesarios para asegurar una adecuada calidad de vida de las personas que más lo necesitan, en particular en unos años marcados por la recesión económica, los resultados son algo mejores que para el caso anterior, pues algo menos de 4.000 residentes en la ciudad de Valencia quedarían a más de 40 minutos de un centro de este tipo y alrededor de 235.000 personas estarían a más de 20 minutos andando. La localización de la oferta de servicios sociales presenta, además, una particularidad: los usuarios se desplazan hasta al centro, pero también los profesionales del mismo deben desplazarse hasta el usuario como parte del servicio. Ello hace muy necesaria la mejora de la accesibilidad a pie, ya que la intervención del trabajador social sobre el territorio resulta fundamental para garantizar los derechos sociales y definir estrategias vinculadas con el bienestar de las personas. En este sentido, resulta preocupante, por ejemplo, que sea tan elevado el número de personas mayores que residen a más de 20 minutos del centro de servicios sociales, o el de extranjeros no comunitarios con esta misma característica.

La situación de la accesibilidad a los centros de servicios sociales de base es especialmente preocupante en el sur de la ciudad, pero también presentan características negativas algunos barrios o áreas del interior de la ciudad. Si consideramos los desplazamientos a pie, es el sur de la ciudad el área que concentra el $73 \%$ de la población a más de 20 minutos del centro de servicios sociales más cercano. Los barrios en peor situación son el Perellonet y una parte de Pinedo y el Saler. En la zona norte se concentra el resto de población afectada, en particular en los barrios de Massarrojos, Benifaraig, Borbotó, Carpesa, Poble Nou, Ciudad Fallera, Norte de Benicalap, Beniferri y Benimamet. Se produce una circunstancia excepcional en cuanto a la accesibilidad a los centros de servicios sociales en Valencia. La mala accesibilidad a pie dibuja una diagonal noroeste-sudeste que cruza la ciudad delimitando un área con mala accesibilidad (en términos de tiempo) a los servicios sociales, a lo largo de barrios tan emblemáticos -y algunos tan demandantes de servicios sociales- como la Vega Baja, L'Amistat, Ciudad Jardín, parte del Cabañal, Beteró, Benimaclet, Patraix, Safranar, Faitanar, Favara y Vara de Quart. Sin embargo, esta franja también incluye distritos como Pla del Real, L’Eixample o Jesús, caracterizados por una renta elevada y que presentan buenas condiciones de accesibilidad. 
Figura 1. Delimitación de áreas de baja accesibilidad a pie (a) y en transporte público (b) a los Centros de Especialidades Médicas en la ciudad de Valencia.

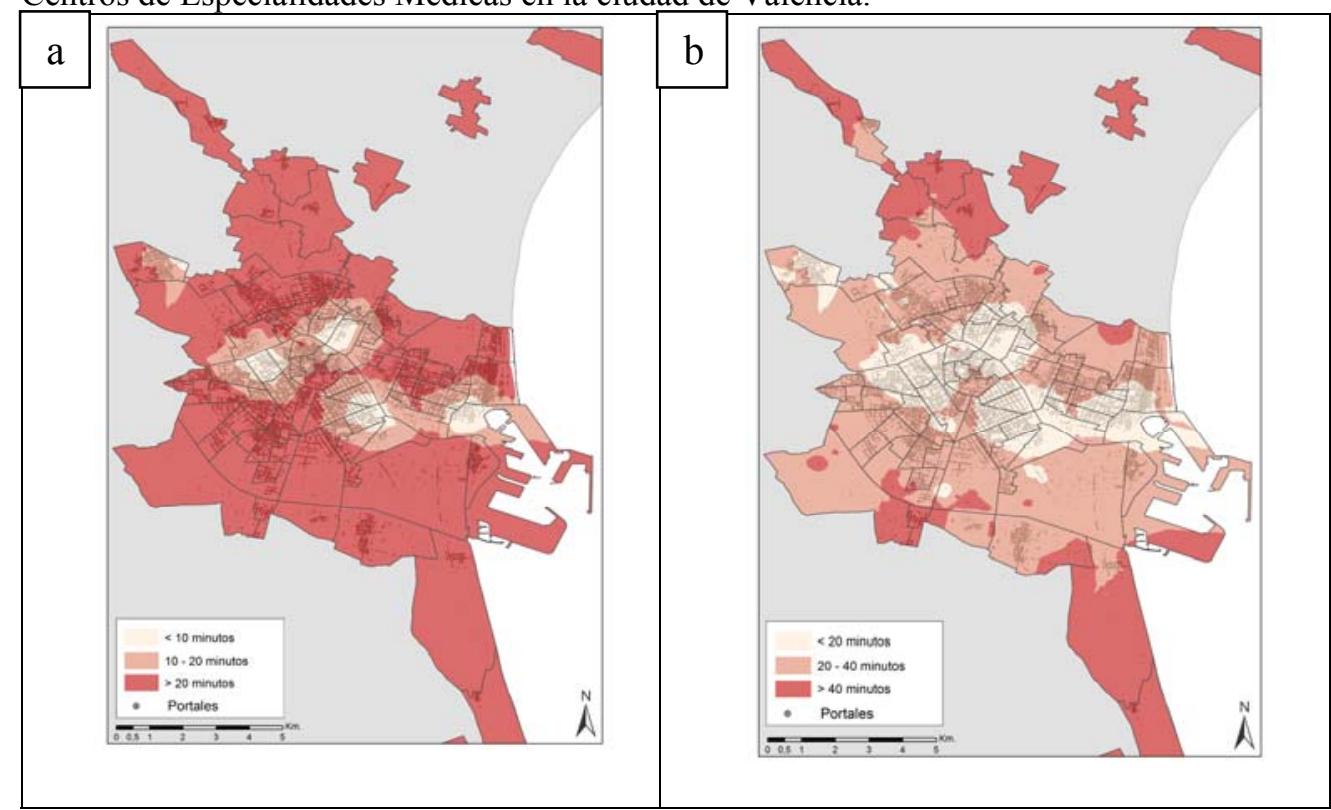

Fuente: Elaboración propia.

Tabla 4: Caracterización de la población en peor situación de accesibilidad a los Centros públicos de Especialidades Médicas. Total de la ciudad de Valencia.

\begin{tabular}{|c|c|c|c|c|}
\hline \multirow{3}{*}{$\begin{array}{l}\text { Población afectada } \\
\text { (Número de personas) }\end{array}$} & \multicolumn{4}{|c|}{ Centros de Especialidades Médicas } \\
\hline & \multicolumn{2}{|c|}{ A pie (a más de 20 minutos) } & \multicolumn{2}{|c|}{ Transporte Público (a más de 40 minutos) } \\
\hline & Número & $\begin{array}{c}\text { Porcentaje sobre el } \\
\text { Total de Valencia }\end{array}$ & Número & $\begin{array}{c}\text { Porcentaje sobre el Total } \\
\text { de Valencia }\end{array}$ \\
\hline Residentes & 443.782 & 59,25 & 14.808 & 1,98 \\
\hline Menores de 16 años & 65.798 & 59,13 & 2.294 & 2,06 \\
\hline Mayores de 65 años & 83.582 & 57,03 & 2.485 & 1,70 \\
\hline $\begin{array}{l}\text { Residentes de origen no } \\
\text { comunitario }\end{array}$ & 38.403 & 60,20 & 852 & 1,34 \\
\hline $\begin{array}{l}\text { Mayores de } 24 \text { años con } \\
\text { nivel de estudios inferior a } \\
\text { Bachillerato }\end{array}$ & 201.491 & 64,63 & 7.113 & 2,28 \\
\hline $\begin{array}{l}\text { Mayores de } 24 \text { años con } \\
\text { nivel de estudios de } \\
\text { Bachillerato o superior }\end{array}$ & 142.296 & 52,70 & 4.280 & 1,59 \\
\hline
\end{tabular}

Fuente: Elaboración propia. 
Figura 2. Delimitación de áreas de baja accesibilidad a pie (a) y en transporte público (b) a los Institutos de Secundaria en la ciudad de Valencia.

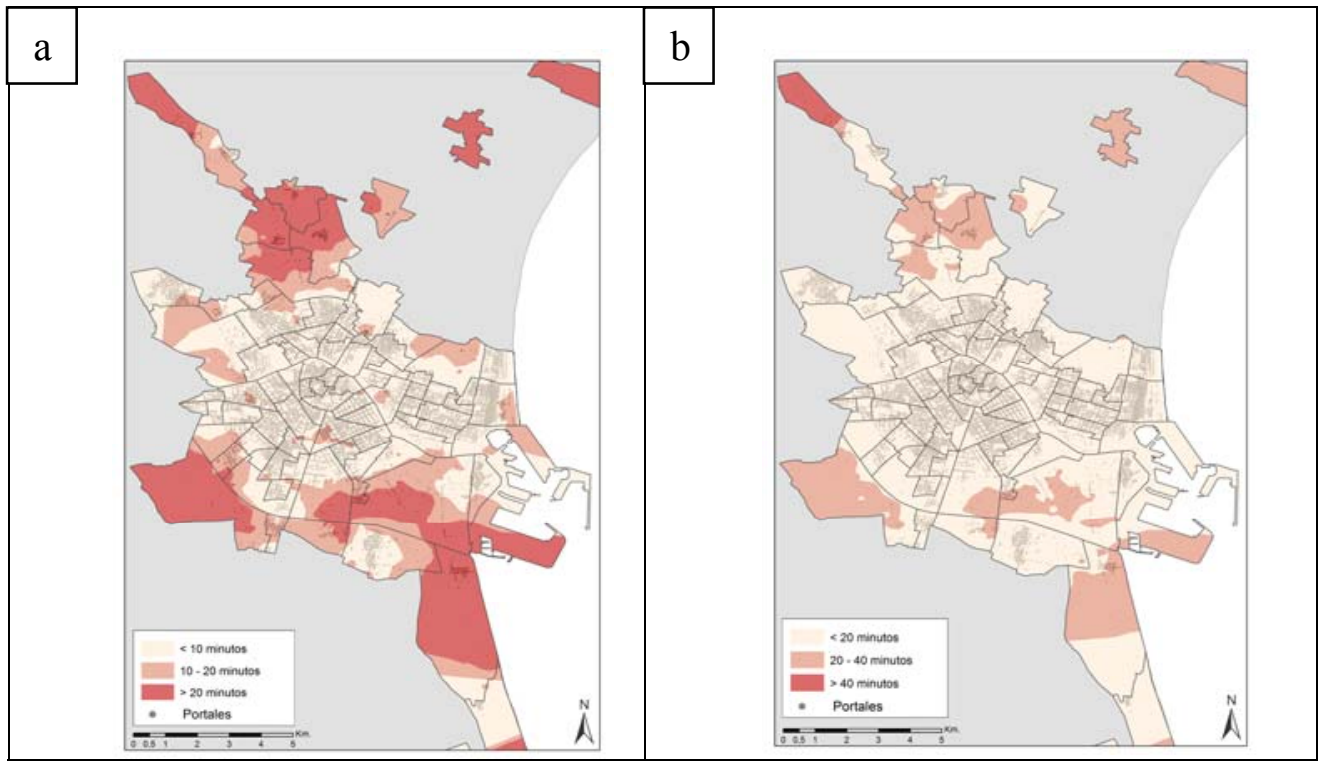

Fuente: Elaboración propia.

Tabla 5. Caracterización de la población en peor situación de accesibilidad a los centros públicos y concertados de Educación Secundaria. Total de la ciudad de Valencia.

\begin{tabular}{|c|c|c|c|c|}
\hline \multirow{3}{*}{$\begin{array}{l}\text { Población afectada } \\
\text { (Número de personas) }\end{array}$} & \multicolumn{4}{|c|}{ Institutos de Educación Secundaria } \\
\hline & \multicolumn{2}{|c|}{ A pie (a más de 20 minutos) } & \multicolumn{2}{|c|}{$\begin{array}{c}\text { Transporte Público (a más de } \\
40 \text { minutos) }\end{array}$} \\
\hline & Número & $\begin{array}{c}\text { Porcentaje sobre } \\
\text { el Total de } \\
\text { Valencia } \\
\end{array}$ & Número & $\begin{array}{c}\text { Porcentaje sobre } \\
\text { el Total de } \\
\text { Valencia } \\
\end{array}$ \\
\hline Residentes & 11.692 & 1,56 & 1.493 & 0,20 \\
\hline Menores de 16 años & 1.747 & 1,57 & 204 & 0,18 \\
\hline Mayores de 65 años & 2.226 & 1,52 & 331 & 0,23 \\
\hline Residentes de origen no comunitario & 369 & 0,58 & 29 & 0,05 \\
\hline $\begin{array}{l}\text { Mayores de } 24 \text { años con nivel de } \\
\text { estudios inferior a Bachillerato }\end{array}$ & 5.739 & 1,84 & 696 & 0,22 \\
\hline $\begin{array}{l}\text { Mayores de } 24 \text { años con nivel de } \\
\text { estudios de Bachillerato o superior }\end{array}$ & 3.317 & 1,23 & 463 & 0,17 \\
\hline
\end{tabular}

Fuente: Elaboración propia. 
Figura 3. Delimitación de áreas de baja accesibilidad a pie (a) y en transporte público (b) a los centros de Servicios Sociales de base en la ciudad de Valencia.

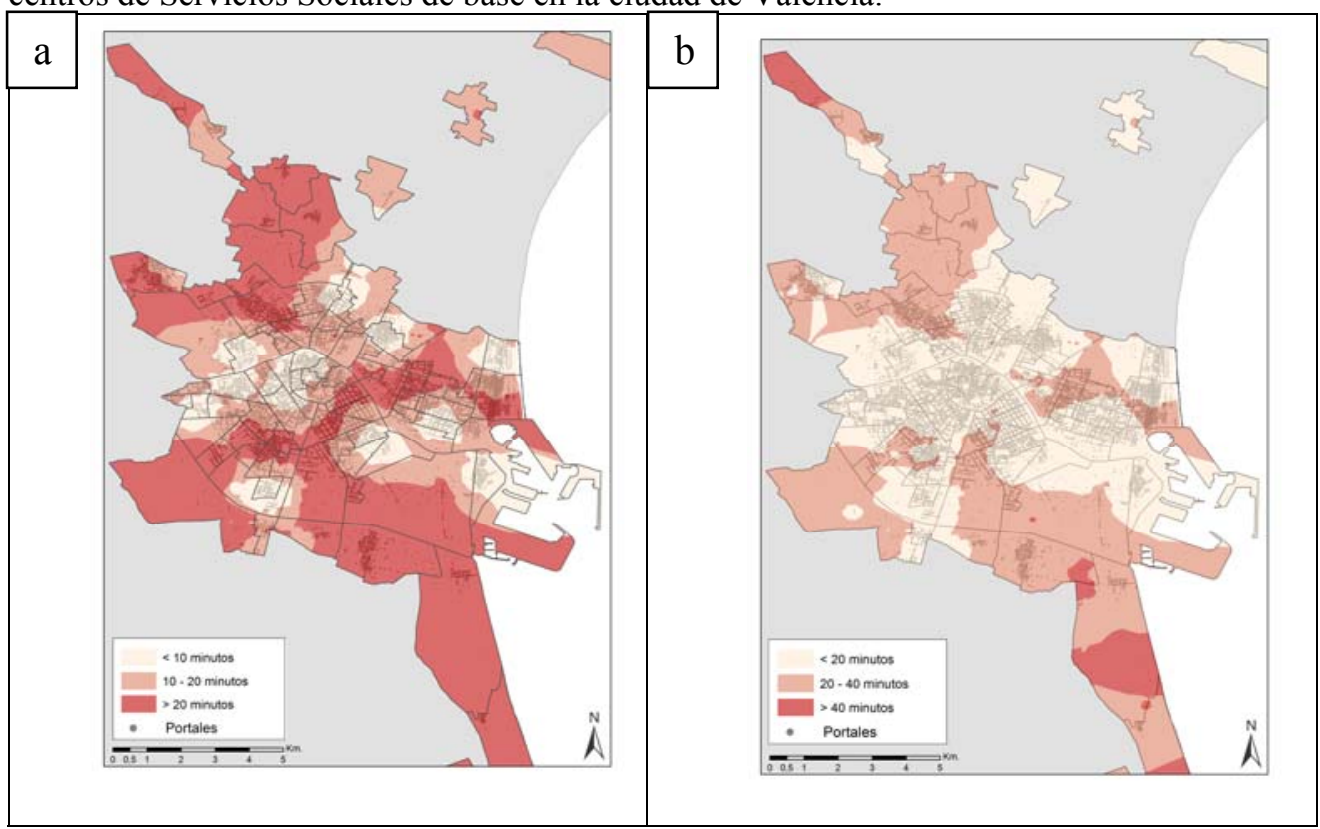

Fuente: Elaboración propia.

Tabla 6. Caracterización de la población en peor situación de accesibilidad a los centros de Servicios Sociales de base. Total de la ciudad de Valencia.

\begin{tabular}{|c|c|c|c|c|}
\hline \multirow{3}{*}{$\begin{array}{l}\text { Población afectada } \\
\text { (Número de personas) }\end{array}$} & \multicolumn{4}{|c|}{ Servicios Sociales de base } \\
\hline & \multicolumn{2}{|c|}{ A pie (a más de 20 minutos) } & \multicolumn{2}{|c|}{$\begin{array}{l}\text { Transporte Público (a más de } \\
40 \text { minutos) }\end{array}$} \\
\hline & Total & $\begin{array}{l}\text { Porcentaje sobre el } \\
\text { Total de Valencia }\end{array}$ & Total & $\begin{array}{c}\text { Porcentaje sobre } \\
\text { el Total de } \\
\text { Valencia }\end{array}$ \\
\hline Residentes & 234.588 & 31,32 & 3.888 & 0,52 \\
\hline Menores de 16 años & 35.903 & 32,27 & 545 & 0,49 \\
\hline Mayores de 65 años & 43.716 & 29,83 & 594 & 0,41 \\
\hline Residentes de origen no comunitario & 18.855 & 29,56 & 68 & 0,11 \\
\hline $\begin{array}{l}\text { Mayores de } 24 \text { años con nivel de } \\
\text { estudios inferior a Bachillerato }\end{array}$ & 98.584 & 31,62 & 1.303 & 0,42 \\
\hline $\begin{array}{l}\text { Mayores de } 24 \text { años con nivel de } \\
\text { estudios de Bachillerato o superior }\end{array}$ & 82.305 & 30,48 & 1.300 & 0,48 \\
\hline
\end{tabular}

Fuente: Elaboración propia. 
Por último, la accesibilidad a los centros de educaciónsecundaria es la mejor de las tres consideradas. Los Institutos de Educación Secundaria, tanto públicos como concertados (la red de centros concertados es muy amplia y se encuentrabien distribuida), parecen ofrecer un buen emplazamiento, dejando fuera de la "cercanía" a menos población que en los dos casos anteriormente descritos; 11.692 personas $(1,5 \%$ del total) quedan a más de 20 minutos a pie, y sólo 1.493 a más de 40 minutos en transporte público. Destaca el elevado número de residentes con sólo estudios básicos (menos que Bachillerato) ubicada a más de 20 minutos andado de un centro de Educación Secundaria, $5.739(1,8 \%)$.

Por lo que respecta a la delimitación de las áreas peor servidas por los centros de educación secundaria, son también los barrios periféricos los más afectados por la falta o lejanía del servicio. El área sur, de nuevo, concentra la mayor parte de la población con peores condiciones de accesibilidad; el $75 \%$ de los afectados (accesibilidad a pie) de la ciudad de Valencia vive en esta zona, que incluye barrios como Pinedo, el Saler, el Palmar, el Perellonet, y una parte de Castellar-Oliveral, de Faitanar y del distrito de Quatre Carreres, en concreto la Punta y Malilla. El 25\% restante reside en la periferia norte, en los barrios antes señalados para el caso de las especialidades médicas. El uso del transporte público mejora significativamente la situación de casi todos estos barrios, excepto en el caso de la periferia más lejana. Sin embargo, la naturaleza del servicio (educativo) desaconseja, salvo excepciones, el uso de cualquier tipo de transporte, debiendo potenciarse y facilitarse la accesibilidad a pie, pues, de todos los servicios analizados, éste es el que exige una mayor cercanía, ya que cabe recordar que una parte de su oferta, la ESO, es obligatoria para los niños de 12 a 16 años.

\subsection{Análisis de la exclusión social y accesibilidad en Valencia}

A lo largo del último decenio, la ciudad de Valencia ha experimentado un empobrecimiento general derivado del impacto de la crisis económica que se ha traducido en importantes aumentos de la tasa de paro y de la población en riesgo de exclusión social. El Área Metropolitana de Valencia, que había mantenido durante el periodo 2001-2008 una evolución del mercado de trabajo más favorable que la media regional, evoluciona peor a partir esta fecha; lo que supone la inversión de una tendencia que se había mantenido durante décadas (Salomy Albertos, 2014). Así, la tasa de paro de la ciudad de Valencia (paro registrado sobre población potencialmente activa) se triplica en 10 años, pasando del 5,5 al 15\% entre 2001 y 2012 (La Caixa, 2013).

Otra importante transformación que impacta sobre la situación social de la ciudad es el aumento de la inmigración extranjera, procedente mayoritariamente de países no comunitarios. Entre 2001 y 2011, los extranjeros pasaron de suponer el 4,2\% de la población de la ciudad de Valencia (algo más de 30.000 personas) a alcanzar el $12,9 \%$ (más de 102.000). Esta población, que se concentró inicialmente en los barrios populares del centro histórico y en algunos otros enclaves dentro del casco urbano 
como Russafa y El Camí Fondo, se descentraliza posteriormente a algunos barrios periféricos y a los municipios más próximos de la corona metropolitana (Torres, 2007).

Tabla 7. Población objeto de intervención prioritaria, afectada por una accesibilidad baja (a pie) y nivel socioeconómico de riesgo.

\begin{tabular}{|c|c|c|c|c|c|c|c|c|c|c|}
\hline & \multicolumn{2}{|c|}{$\begin{array}{l}\text { Total de } \\
\text { población }\end{array}$} & \multicolumn{2}{|c|}{$\begin{array}{l}\text { Total mayores } \\
\qquad \text { de } 65\end{array}$} & \multicolumn{2}{|c|}{$\begin{array}{l}\text { Total menores de } \\
16 \text { años }\end{array}$} & \multicolumn{2}{|c|}{$\begin{array}{c}\text { Residentes de } \\
\text { origen no } \\
\text { comunitario }\end{array}$} & \multicolumn{2}{|c|}{$\begin{array}{c}\text { Mayores de } 24 \\
\text { años con nivel de } \\
\text { estudios inferior } \\
\text { a Bachillerato }\end{array}$} \\
\hline & Total & $\%$ & Total & $\%$ & Total & $\%$ & Total & $\%$ & Total & $\%$ \\
\hline $\begin{array}{l}\text { Centros de Esp. } \\
\text { Médicas }\end{array}$ & 143.484 & 19,15 & 27.749 & 18,9 & 21.270 & 19,11 & 13.675 & 21,44 & 79.769 & 25,58 \\
\hline $\begin{array}{l}\text { Institutos de } \\
\text { Educ. Secund. }\end{array}$ & 5.499 & 0,73 & 1.200 & 0,82 & 774 & 0,7 & 174 & 0,27 & 3.167 & 1,02 \\
\hline $\begin{array}{l}\text { Centros de S. } \\
\text { Sociales }\end{array}$ & 50.894 & 6,79 & 9.565 & 6,53 & 7.946 & 7,14 & 3.727 & 5,84 & 27.113 & 8,70 \\
\hline Población Total & 749.095 & 100 & 146.552 & 100 & 111.282 & 100 & 63.791 & 100 & 311.787 & 100 \\
\hline
\end{tabular}

Fuente: Elaboración propia

Figura 4. Nivel socioeconómico (Componente 1 ACP) y accesibilidad a pie a los Centros de Especialidades Médicas y zonas de intervención prioritaria.

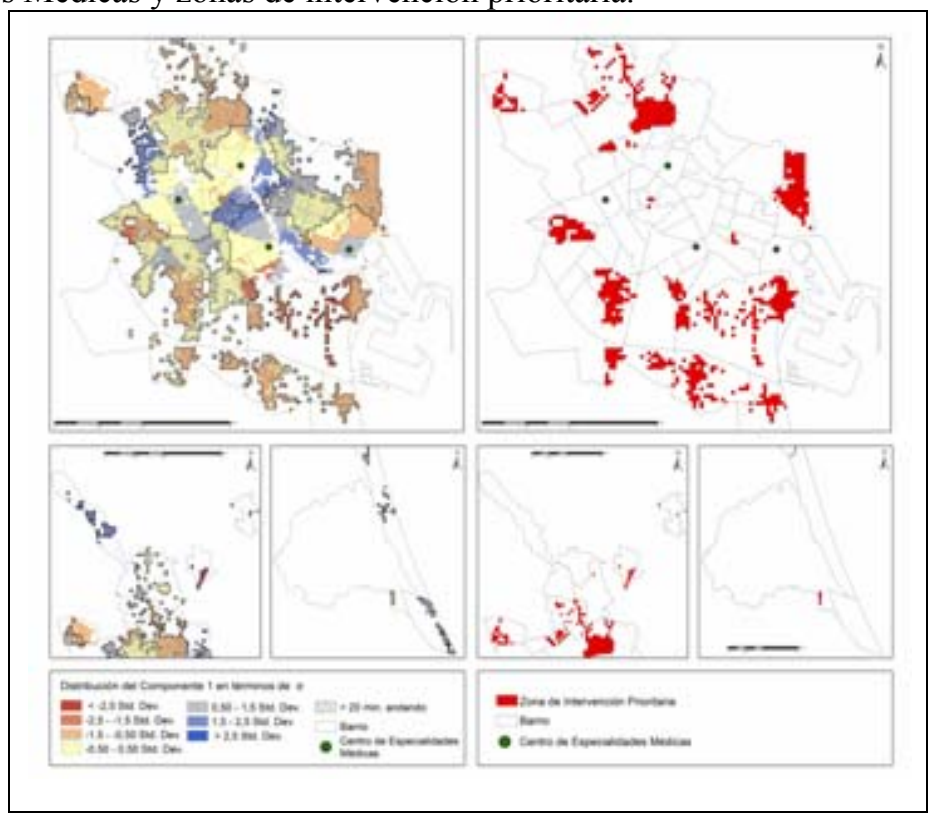

Fuente: Elaboración propia 
La cuestión que se plantea tras el análisis de la accesibilidad a los servicios de proximidad considerados, es si los barrios o áreas de la ciudad con peor accesibilidad resultan ser también barrios con problemas socioeconómicos. En tal caso, la mejora de las condiciones de vida de la población en estas zonas debería ser objeto de acción política preferente. Para tal fin es necesario caracterizar el nivel socioeconómico de las distintas zonas de la ciudad y relacionarlo con su nivel de accesibilidad.Ya que no se cuenta con datos sobre renta per cápita para la ciudad de Valencia a nivel de barrio, la combinación de una serie de variables (ya señaladas) que de manera indirecta nos indiquen el nivel de renta de la población, es una forma de acercarnos a la realidad económica a nivel micro.Para ello, el método ACP nos ayuda a determinar cuáles son las variables, relacionadas entre sí, que caracterizan las distintas áreas o barrios de la ciudad. Se trata de un método estadístico básico pero muy eficaz para agrupar dichas variables que identifican los territorios de análisis. La combinación entre el componente 1 y los valores de la accesibilidad (figuras 4, 5 y 6 ) nos permite detectar aquellas zonas en las que coinciden una mayor lejanía al servicio (más de 20 minutos andando) y unas condiciones socioeconómicas menos favorables. Se trata de lo que podríamos denominar espacios de intervención prioritaria.

De acuerdo con ambos indicadores, los barrios localizados en el centro y en el primer ensanche de la ciudad son los que presentan mejor situación, tanto desde el punto de vista socioeconómico como de accesibilidad a los servicios, que se encuentran ubicados a corta distancia, tanto para desplazamientos a pie como en transporte público. Por el contrario, las áreas más desfavorecidas son las localizadas en la periferia, que en ocasiones se encuentran más cercanas físicamente a los servicios ofertados por otros municipios lindantes que a los de la misma capital. A este respecto, una parte importante de la periferia norte y sur se encuentra en condiciones de desventaja con respecto a otros barrios con similar situación económica donde la accesibilidad a los servicios públicos no es un problema.

Una vez detectadas las áreas más desfavorecidas, es posible, a partir de la información del Padrón desagregada a nivel de portal, determinar con detalle cuánta población reside en dichas áreas y cuáles son sus características básicas (tabla 7). En la ciudad de Valencia, el 19\% de la población (todos ellos residentes en áreas de renta media y baja) se beneficiaría de una mejora en la accesibilidad a los centros de especialidades médicas; concretamente, el $21,4 \%$ de los residentes no comunitarios y el $25,5 \%$ de la población con nivel de estudios bajo. En el caso de los servicios educativos, la situación es mejor, pero un total de 5.499 personas residentes en áreas desfavorecidas se encuentran alejados de los centros de educación secundaria, suponiendo el $0,7 \%$ del total de la población residente en la ciudad. Sin embargo, el porcentaje aumenta hasta el 1,02 cuando se considera a los residentes con estudios sólo elementales. Por último, el $6,79 \%$ de la población se encuentra alejada de los centros de servicios sociales de base y, a su vez, reside en áreas desfavorecidas. La cifra aumenta ligeramente si se considera a la población menor de 16 años $(7,14 \%) \mathrm{y}$, sobre todo, a los que cuentan con niveles de cualificación bajo $(8,7 \%)$. 
Figura 5. Nivel socioeconómico (Componente 1 ACP) y accesibilidad a pie a los IIEs y zonas de intervención prioritaria.

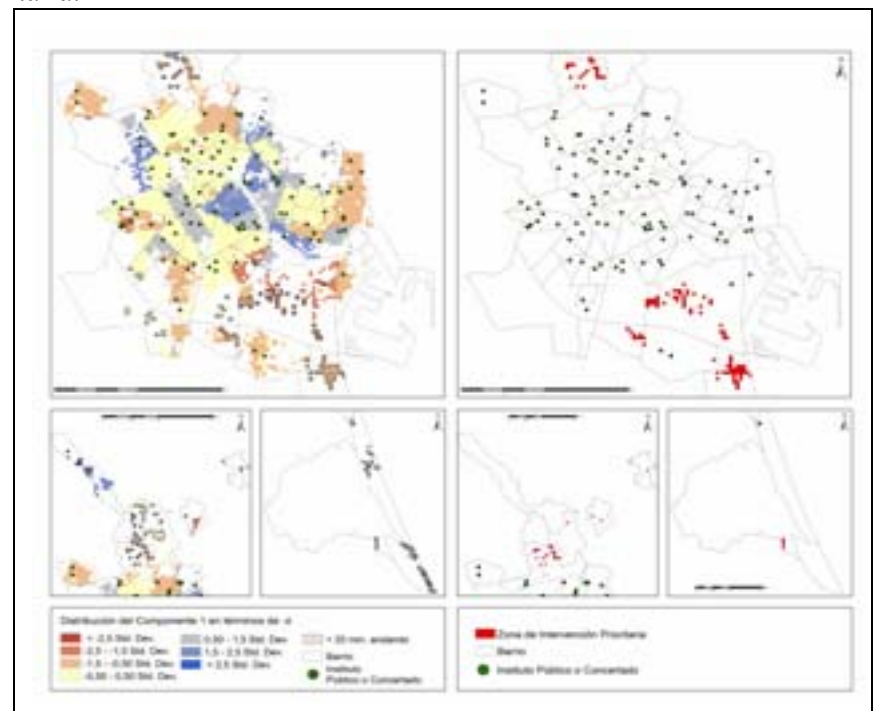

Fuente: Elaboración propia

Figura 6. Nivel socioeconómico (Componente 1 ACP) y accesibilidad a pie a los Centros de Servicios Sociales y zonas de intervención prioritaria.

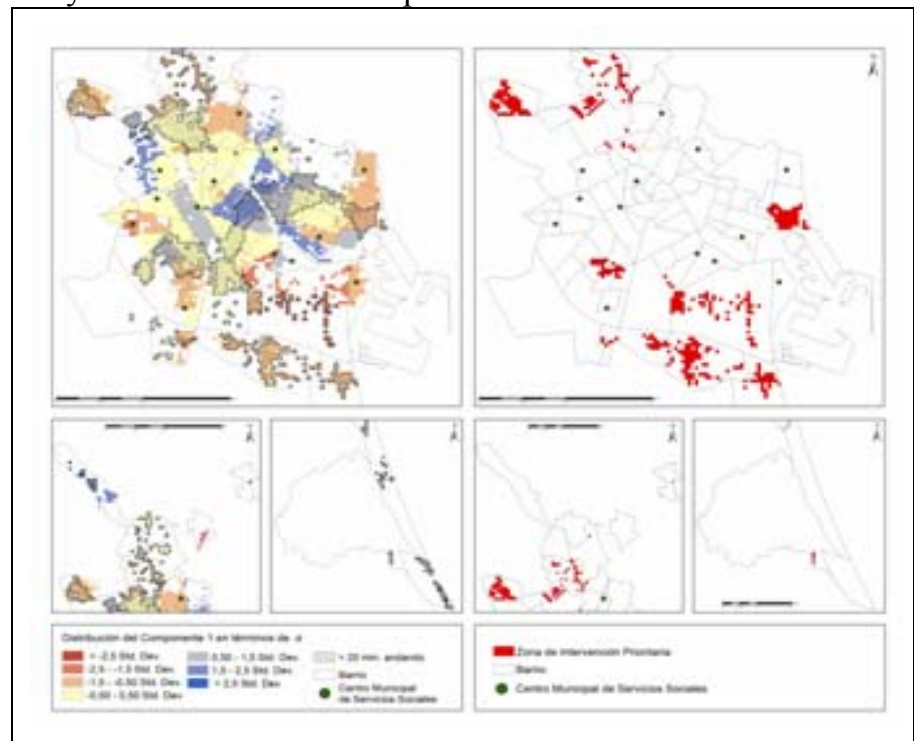

Fuente: Elaboración propia 
En conclusión, y aunque es evidente que toda la población, sea cual sea su nivel de renta, debe disfrutar de una buena accesibilidad (cercanía) a los servicios públicos de proximidad, la realidad es que en una situación de recesión económica y contención del gasto, es necesario priorizar las inversiones y orientarlas allí donde éstas son más urgentes o necesarias. El análisis realizado ayuda, en este sentido, a la toma de decisiones públicas.

\section{Conclusiones}

Las dinámicas socioeconómicas recientes, y en particular la intensificación de las corrientes migratorias y los efectos de la crisis económica, han tendido a agravar las desigualdades en el interior de los espacios metropolitanos. Esta tendencia, general en las ciudades españolas, ha afectado también al Área Metropolitana de Valencia (Salom y Fajardo, 2017). La concentración de los grupos de bajas rentas en determinadas áreas en el interior de las ciudades agrava su situación al generarse dinámicas de segregación territorial. Las políticas públicas de provisión de servicios básicos pueden contribuir a atenuar los efectos negativos de estos procesos. Sin embargo, en un contexto de recortes presupuestarios generalizado, la planificación de los equipamientos públicos debe realizarse de la forma más depurada posible para maximizar el impacto social de la inversión realizada. La metodología propuesta en esta investigación y aplicada al caso de la ciudad de Valencia permite avanzar en la investigación aplicada sobre niveles de pobreza y riesgo de marginación en el interior de la ciudad. La accesibilidad a los servicios, el uso de SIG y la combinación con la información sociodemográfica a nivel de portal, facilitan un análisis de mayor detalle, permitiendo la detección de áreas frágiles o peor servidas, todo lo cual ayuda a la toma de decisiones políticas.

Aunque en la ciudad de Valenciano se detectan barrios en riesgo elevado, la metodología aplicada ha permitido delimitar una serie de áreas que requieren especial atención, en particular ubicadas en la periferia más exterior de la ciudad -norte y sur, caracterizadas por un poblamiento disperso, escaso y con dificultades de acceso a los equipamientos básicos, especialmente a los sanitarios y de servicios sociales. También áreas concretas de barrios centrales sufren malas condiciones de accesibilidad. La detección de estas zonas resulta importante para planificar cualquier tipo de intervención. En conclusión, la reducción de las desigualdades sociales en la ciudad de Valencia requiere desarrollar las infraestructuras adecuadas para servir a las áreas más alejadas del centro y garantizar su acceso a los servicios de proximidad, en particular a las personas que más lo necesitan. Los estudios realizados en esta línea están resultando, pues, de enorme importancia para generar información útil para la toma de decisiones. 


\section{Bibliografía}

Albertos, J.M. (2014): La movilidad sostenible en áreas metropolitanas. Evolución reciente y escenarios de futuro en el Área Metropolitana de Valencia, en Pitarch, M.D. (ed.), Sostenibilidad en áreas metropolitanas, 63-80.

Alguacil, J.; Camacho, J. y Hernández Aja, A. (2014): La vulnerabilidad urbana en España. Identificación y evolución de los barrios vulnerables, EMPIRIA. Revista de Metodología de Ciencias Sociales, 27, 73-94.

Andreotti, A., Mingione, E., y Polizzi, E. (2012). Local welfare systems: a challenge for social cohesion. Urbanstudies, 49(9), 1925-1940.

Bauman, Z. (2005): Identidad. Madrid: Losada.

Benko, G. y Lipietz, A. (1994): Las regiones que ganan. Distritos y redes: los nuevos paradigmas de la Geografía Económica. Institución Alfons el Magnànim, Valencia.

Bhat, Ch., et al. (2000): Development of an Urban Accessibility Index: Literature Review, Centre of Transportation Research, Austin, University of Texas.

Blockland, T. et al. (eds.) (2016): Creating the unequal city. The exclusion consequences of everyday routines in Berlin, Routledge, Nueva York.

Calvo, J.L. et al. (2001): Análisis, diagnóstico y ordenación de equipamientos mediante formulaciones cartografiables: valoración de la accesibilidad y requerimientos de la asistencia hospitalaria en la CC.AA de la Rioja mediante la técnica de potenciales, Berceo, n. 141, 247-268.

Cañada Torrecilla, R. (2007): Técnicas de interpolación espacial deterministas y exactas: media ponderada por el inverso de la distancia y funciones de base radial, en Moreno Jiménez, A. (Coord.): Sistemas y análisis de la información geográfica. Madrid, Ra-Ma, 781-809

Cerdá, J. (2014): El uso del tiempo en espacios próximos. Una caracterización de la ciudad de Barcelona, Ciudades, n. 17, 65-97

Fernández Durán, R. (1993): La explosión del desorden. La metrópoli como espacio de la crisis global, Ed. Fundamentos, Madrid.

Foguet, J. M. (1989):Análisis multivariante: análisis de componentes principales. Editorial Hispano Europea S.A., Colección ESADE, Barcelona, España.

Gajai Diaz, F. (2016): València, del boom al crac (1979-2007), en València, 1808-2015: la història continua... Balandra Edicions, 187-205

Garrocho, C. y Campos J. (2006): Un indicador de accesibilidad a unidades de servicios clave para ciudades mexicanas: fundamentos, diseño y aplicación, Economía, Sociedad y Territorio, vol. VI, n. 22, 1-60.

Harvey, D. (2012): Revel cities: from the right to the city to the urban revolution. Londres: Verso.

Hernández Aja, A. (2000): La ciudad estructurada, Textos sobre Sostenibilidad, Cuadernos sobre Investigación Urbanística, 42, 13-27.

Hudson, R. (2013): Thatcherism and its geographical legacies: the new map of socio-spatial inequality in the Divided Kingdom, The Geographical Journal, 179 (4), 377-381. 
La Caixa (2013): Anuario Económico de España, 2013, www.anuarioeco.lacaixa.comunicacions.com (consulta realizada 4 febrero, 2017)

López-Roldán, P.; Fachelli, S. (2016). Análisis factorial.En P. López-Roldán y S. Fachelli, Metodología de la Investigación Social Cuantitativa. Bellaterra (Cerdanyola del Vallès): Dipòsit Digital de Documents, Universitat Autònoma de Barcelona. $1^{\mathrm{a}}$ edición, versión 3. Edición digital: http://ddd.uab.cat/record/142928

Marquet, S. y Miralles, C. (2014): La proximidad en Barcelona. Un análisis desde los tiempos de desplazamiento cotidianos, Ciudades, $\mathrm{n}^{\circ} 17,99-120$

Monfort Mir, V.M. (2010): Valencia, turismo cubista, en Sorribes, J. (ed.), Valencia, 19572007, 101-154

Nel.lo, O. (2007): Contra la dispersión, intensidad. Contra la segregación, ciudad, en Indovina, F. (coord.), La ciudad de baja densidad, Serie Territorio, Diputación de Barcelona, Barcelona.

Pitarch-Garrido, M.D. (2013): Measuring Equity and social sustainability through accessibility to public services by public transport. The case of the Metropolian area of Valencia (Spain), European Journal of Geography, 4(1), 64 - 85.

Pitarch-Garrido, M.D. (2018): Social Sustainability in Metropolitan Areas: accessibility and Equity in the Case of the Metropolitan Area of Valencia (Spain). Sustainability, 10, 371.

Rawls, J. (2001): Justice as Fairness. Cambridge. Harvard University Press.

Salom J. y Pitarch, M.D. (2017): Análisis del impacto en el turismo de la estrategia de desarrollo urbano basada en megaproyectos. El caso de la ciudad de Valencia, Cuadernos de Turismo, 40, 573-598

Salom, J. y Albertos, J.M. (2014): La crisis económica en los distritos industriales valencianos, en Albertos, J.M. y Sánchez, J.L. (coords.) Geografía de la crisis económica en España, PUV-Publicaciones de la Universidad de Valencia, Valencia, 467-495.

Salom, J. y Fajardo, F. (2015): El Área metropolitana de Valencia en el sistema global de ciudades: El impacto de la crisis económica en la red de relaciones financieras de las empresas multinacionales, en de la Riva, J., Ibarra, P., Montorio, R., Rodrigues, M. (Eds.) 2015: Análisis espacial y representación geográfica: innovación y aplicación, Universidad de Zaragoza-AGE, http://congresoage.unizar.es/eBook/trabajos/227_Salom.pdf

Salom, J. y Fajardo, F. (2017): Cambios recientes en la estructura territorial sociodemográfica del área metropolitana de Valencia (2001-2011), Boletín de la Asociación de Geógrafos Españoles, 73, 123-147.

Sánchez Hernández, J.L. (2003): Naturaleza, localización y sociedad. Tres enfoques para la Geografía Económica, Ediciones Universidad de Salamanca, Salamanca.

Santamarina, B. y Moncusí, A. (2013): «De huertas y barracas a galaxias faraónicas. Percepciones sociales sobre la mutación de la ciudad de Valencia», Papers: Revista de Sociologia, 98(2), pp. 365-391

Sassen, S. (1991): The Global City: New York, London, Tokyo. Princeton, N.J.: Princeton University Press.

Sassen, S. (1998): Ciudades en la economía global: enfoques teóricos y metodológicos, Eure, v. 24(71), 1-21. 
Shelton, T. et al. (2015): Social media and the city: rethinking urban socio-spatial inequality using user-generated geographic information, Landscape and urban planning, 142, 198211.

Subirats, J. (ed.) (2005): Análisis de los factores de exclusión social, Doc. De trabajo, n. 4, Fundación BBVA, Generalitat de Catalunya, Barcelona.

Torres Pérez, F. (2007): Nous veïns a la ciutat. Els inmigrants a València i Russafa, PUV, Valencia

Werna, E. (2001): Combating urban inequalities. Challenges for managing cities in the developing world, Edward Elgar Publ., Londres. 